\title{
Experimental study of thermoelastic damping in MEMS gyros
}

\author{
Amy Duwel, John Gorman, Marcie Weinstein, Jeff Borenstein, Paul Ward
}

The Draper Laboratories, MS 37, 555 Technology Square, Cambridge, MA 02138, USA

Massachusetts Institute of Technology, Cambridge, MA, USA

\begin{abstract}
We present new experimental data illustrating the importance of thermoelastic damping (TED) in MEMS resonant sensors. MEMS gyroscopes have been used to demonstrate that both the choice of materials and variations in device design can lead to significant differences in the measured quality $(Q)$ factors of the device. These differences in the $Q$-factor can be explained by including the contribution of thermoelastic damping, which varies strongly between the different silicon etch-stop compositions used in this study. Known damping mechanisms, such as fluid damping, anchor damping, and electronics damping are minimized and held fixed in this experiment so that materials effects can be isolated.
\end{abstract}

Keywords: Quality factor; Damping; $Q$; Thermoelastic

\section{Introduction}

Thermoelastic damping (TED) has been identified as an important loss mechanism in MEMS resonators [1-3]. The impact of device geometry on the level of damping has been considered both experimentally and theoretically [3-5]. Although most work till date has focused on analytically tractable beam systems, the need for high $Q$ resonators extends to a wide variety of applications, including accelerometers [6], Coriolis rate sensors [7], chemical sensors [8], and RF filters [9]. In most applications, maintaining a high quality factor results in reduced readout errors, lower power requirements, improved stability, and increased sensitivity.

The resonator devices used in this study were MEMS gyros fabricated at Draper Laboratory. The excitation and readout mechanism is capacitive, and the measurements are made in a vacuum better than $1 \mathrm{mTorr}$. We find that the simple calculation by Zener [1,2] for thermoelastic damping in flexural mode beam resonators work well to describe the effects of beam width and material properties on the $Q$-factor of the resonant-mode gyros. In this paper, the effects of alloying on thermal conductivity and the resulting effect on $Q$-factor are explored. We compare the measurements on devices made from silicon-germanium alloys with
Ge concentrations of up to $30 \%$, and with boron doping of up to $2 \times 10^{20} \mathrm{~cm}^{-3}$. Using thermal conductivity data from Dismukes et al. [10] on Boron doped SiGe materials, we show that the differences in thermal conductivity can account for our observed variations in $Q$ factors.

In Section 2, we introduce the MEMS resonator being studied and the experimental setup. We also discuss the impact of our measurement setup on the measured $Q$ of the resonator. In Section 3, we review the thermoelastic damping and discuss some of the important material parameters that must be known to quantify this effect. Section 4 compares a theory for total $Q$-factor to measured data.

\section{Experimental setup}

The MEMS gyro is a tuning fork resonator which senses the angular rate. A photograph of the gyro (top view) is shown in Fig. 1. The masses are driven in a tuning fork resonance mode in the plane of the wafer. In response to a $\hat{z}$-directed angular rate, the two proof masses move out of the wafer plane $( \pm \hat{y})$ in opposite directions. This motion is sensed capacitively and the amplitude of this motion is the desired signal proportional to angular rate. The resonating proof mass always serves as one plate of the sense capacitor, while the other plate is fixed to the substrate. The gyro has two possible readouts - one fixed capacitor plate is beneath the proof mass to sense $y$-motion, and the other senses the tuning fork motion in the $x$-direction. In this experiment, we focus on measurements of the tuning fork 


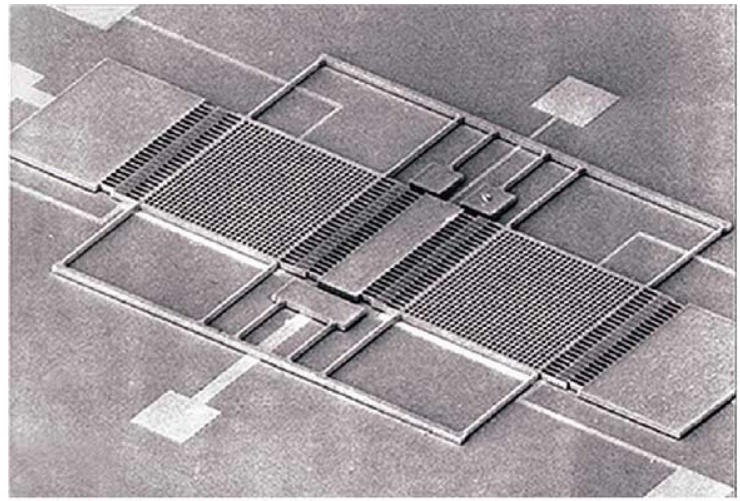

Fig. 1. Photograph of a Draper tuning fork gyro.

resonance in the $x$-direction for comparing the $Q$ values of devices made from various materials.

In the design of resonant sensors, the readout circuit must balance the need for low insertion loss with high $Q$-factor. Any output signal current, for example, results from a coupling to the resonating sensor and can lead to dissipation of the mechanical resonance [11]. In the presence of a voltage bias, the current is defined by $I=\dot{C} V+\dot{V} C$, and for the case of a dc bias is simply proportional to the proof mass motion through $\dot{C}=\dot{u} \mathrm{~d} C / \mathrm{d} u$, where $u$ is the amplitude of motion in the $x$-direction. This current is amplified and converted to a voltage, and the transfer function of the output to the input voltage is used to measure $Q$. Fig. 2 shows the circuit schematic used to drive the sensor and measure the $Q$-value. For $Q$ measurements, the input drive voltage is the sum:

$V_{\text {drive }}=V_{\mathrm{dc}}+V_{\text {in }} \sin \omega t$,

so that the force on the sensor is:

$F_{\text {drive }}=V_{\mathrm{dc}}^{2}+V_{\mathrm{dc}} V_{\text {in }} \sin \omega t+V_{\text {in }}^{2} \sin ^{2} \omega t$

The transfer function is taken by comparing $V_{\text {out }}$ at $\omega$ to $V_{\text {in }}$.

We use the half power bandwidth relative to the resonant frequency to measure the $Q$. This works well as long as the drive coupling to other modes is minimized and the

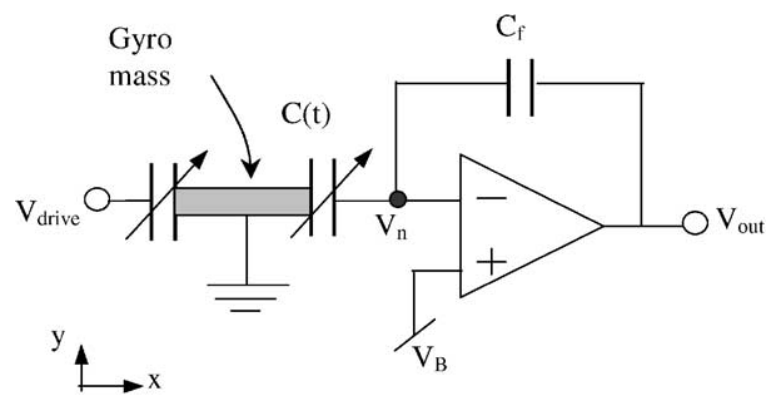

Fig. 2. Circuit schematic of capacitive readout for MEMS resonator. The mode of interest results in a movement of the gyro mass in the $x-z$ plane. The gyro resonance is excited on one side of the mass by an electrostatic force and the change in the readout capacitance is sensed. Comb finger capacitors are used so that the readout is linear in $x$.

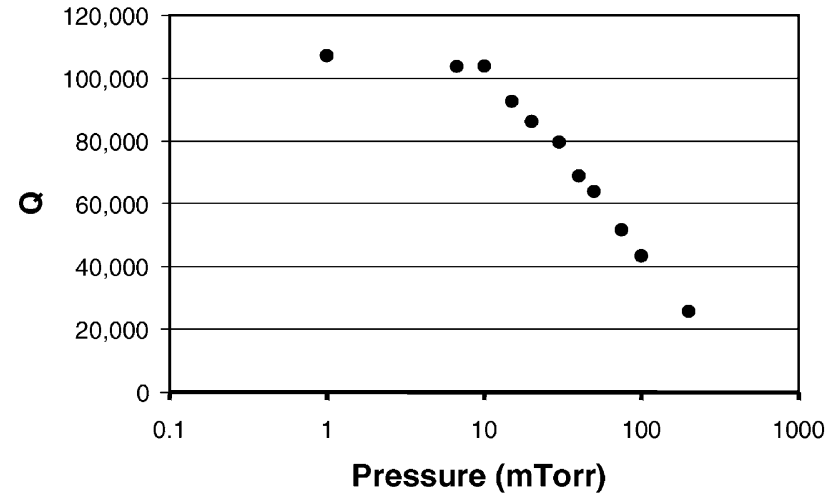

Fig. 3. Pressure versus measured $Q$-value of a MEMS gyro. The measurements are taken to verify that the influence of gas damping is minimized at $1 \mathrm{mTorr}$.

spectrum looks like that of an isolated resonator. When using this method, it is also important to drive the resonator at low amplitudes, so that nonlinear effects are negligible. Bandwidth and ringdown testing ${ }^{1}$ typically agreed to better than $10 \%$ even without taking the different bias conditions and corresponding electrostatic spring effects into account. The resonance peaks were very symmetric, and the standard deviation of the repeated bandwidth measurements is $1 \%$.

All measurements were conducted at the wafer level under a vacuum of approximately $1 \mathrm{mTorr}$. Fig. 3 shows measurements of $Q$-factor as the pressure in the wafer probe station is reduced. In the range of 1 mTorr, the contribution of fluidic damping is reduced so that measurements of $Q$ are found to be independent of small changes in vacuum level. We expect any residual fluidic damping to be constant for all measured devices due to similar device geometry.

The measurement itself can influence the quality factor of the gyro. In order to produce a signal out, power has to be coupled from the resonator, and this power can be dissipated. We include in the Appendix A, a simple model for the gyro dynamics and the role of the applied voltages. We use this model to calculate the electronics contribution to the $Q$-factor and include it in the total calculated $Q$. Since all of our gyro designs use almost identical input and output coupling capacitors, the contribution of the electronics damping does not vary significantly from device to device.

\section{Thermoelastic damping}

Thermoelastic damping arises from the coupling of the stress-strain state equation to heat flow in the material. The familiar effects of the coefficient of thermal expansion $(\alpha)$,

\footnotetext{
${ }^{1}$ Note that both bandwidth and ringdown tests for measuring $Q$ will be affected by the presence of nearby modes. According to theory (Nayfeh), ringdown tests should not be affected by amplitude nonlinearities. For small drive amplitudes, the effects of amplitude nonlinearities on the resonance curve measurements can also be minimized.
} 
where stresses $(\sigma)$ and strains $(\varepsilon)$ can be thermally induced, can be derived from the dependence of the Hemholtz free energy on both strain and temperature. From this starting point, however, one also finds that the temperature gradient in a material can be driven by changes in the strain. The coupled (linearized) state equations for an isotropic medium are $[12,13]$ :

$\varepsilon=\frac{\sigma}{E}+\alpha T$

$\frac{\mathrm{d} T}{\mathrm{~d} t}=\frac{\kappa}{C_{\mathrm{V}}} \nabla^{2} T-\frac{E \alpha T_{0}}{C_{\mathrm{V}}} \frac{\mathrm{d} \varepsilon}{\mathrm{d} t}$

where $E$ is the Young's modulus (Poisson's effect is neglected here), $T_{0}$ the nominal equilibrium temperature, and $T$ the temperature offset from equilibrium. The coefficient of thermal expansion is denoted by $\alpha$, the thermal conductivity by $\kappa$, and the heat capacity at constant volume by $C_{\mathrm{V}}$.

An effective transfer function for stress versus strain can be found by applying Fourier transform techniques to (3) and (4). By assuming sinusoidal steady-state solutions with periodic or discrete spatial dependence $[\varepsilon(t, x) \Leftrightarrow \tilde{\varepsilon}(s, k)]$, temperature can be eliminated in (3). This yields a complex, frequency dependent Young's modulus that includes both mechanical and thermal material properties [14]. This effective modulus exhibits a zero followed closely by a pole in the frequency domain, which results in a resonance (called a Debye peak) in the imaginary component. With a complex modulus, the strain in the material can be out of phase with the applied sinusoidal stress, and the integral of work done (energy lost per cycle) will be a function of the phase shift.

The ratio of the imaginary component to the real component of the modulus defines a frequency dependant quality factor for the material. This approach reproduces with the quality factor derived by Zener [1,2] for a flexural mode beam resonator:

$Q_{\mathrm{TED}}^{-1}=\frac{E \alpha^{2} T_{0}}{C_{\mathrm{V}}} \frac{\omega \tau}{1+(\omega \tau)^{2}}$

with

$\tau=\frac{C_{\mathrm{V}} b^{2}}{\kappa \pi^{2}}$

where $\omega$ is the mechanical resonance frequency of the beam and $b$ represents the width of the resonator in the plane of the resonance motion. In deriving expression (5) from (3) and (4) using Fourier transforms, a spatial dependence, such as $k=\pi / b$ (the first eigen value of the uncoupled thermal equation of a flexural mode beam) must be applied, which is analogous to the type of approximation used by Zener [1,2].

While this approach does not offer a rigorous solution to the problem (which can be found in [4]), it does connect thermoelastic effects with standard inelastic damping theory and offers intuition for the loss mechanism. Different material relaxation mechanisms, such as point defect motion, dislocation movement, and thermoelastic damping will each have an associated relaxation time constant, as in Eq. (6). The total amount of damping will depend on the proximity of the device operating frequency to the Debye peak frequencies.

In the resonators tested, several different materials were used. We especially focused on Boron-doped SiGe epitaxial materials since the presence of Ge reduces strain in the epi layer and the resulting devices can be extremely flat and well-machined. In addition, the use of $\mathrm{Ge}$ as an etch stop eliminates the need for EDP in processing [15], which is a huge advantage. However, considering the issue of thermoelastic damping, the use of an alloy can significantly reduce the thermal conductivity due to phonon scattering. Based on the data of Dismukes [10], we summarize in Table 1 the

Table 1

Material and device parameters used in thermoelastic damping calculations

\begin{tabular}{|c|c|c|c|c|c|c|c|c|c|}
\hline Quantity & Symbol & Units & $\mathrm{SiB}$ epi & $\mathrm{SiGeB} 2 \%$ & SiGeB $23 \%$ & SiGeB $30 \%$ & B-diff & Si value & Ge value \\
\hline $\begin{array}{l}\text { Thermal coefficient } \\
\text { of expansion }\end{array}$ & $\alpha$ & $1 /{ }^{\circ} \mathrm{C}$ & $2.57 \mathrm{E}-06$ & $2.65 \mathrm{E}-06$ & $3.33 \mathrm{E}-06$ & $3.55 \mathrm{E}-06$ & $2.59 \mathrm{E}-06$ & $2.59 \mathrm{E}-06$ & $5.80 \mathrm{E}-06$ \\
\hline Reference & & & Si value & Int & Int & Int & [20] & {$[17,18]$} & [18] \\
\hline $\begin{array}{l}\text { Modulus } \\
\text { Reference }\end{array}$ & $E$ & $\mathrm{~N} / \mathrm{m}^{2}$ & $\begin{array}{l}1.69 \mathrm{E}+11 \\
\text { Si value }\end{array}$ & $\begin{array}{l}1.68 \mathrm{E}+11 \\
\text { Int }\end{array}$ & $\begin{array}{l}1.54 \mathrm{E}+11 \\
\text { Int }\end{array}$ & $\begin{array}{l}1.49 \mathrm{E}+11 \\
\text { Int }\end{array}$ & $\begin{array}{l}169 \mathrm{E}+11 \\
\mathrm{Si} \text { value }\end{array}$ & $\begin{array}{l}1.69 \mathrm{E}+11 \\
{[18]}\end{array}$ & $\begin{array}{l}1.03 \mathrm{E}+11 \\
{[18]}\end{array}$ \\
\hline $\begin{array}{l}\text { Density } \\
\text { Reference }\end{array}$ & $\rho$ & $\mathrm{kg} / \mathrm{m}^{3}$ & $\begin{array}{l}2.33 \mathrm{E}+03 \\
\mathrm{Si} \text { value }\end{array}$ & $\begin{array}{l}2.39 \mathrm{E}+03 \\
\text { Int }\end{array}$ & $\begin{array}{l}3.02 \mathrm{E}+03 \\
\text { Int }\end{array}$ & $\begin{array}{l}3.24 \mathrm{E}+03 \\
\text { Int }\end{array}$ & $\begin{array}{l}2.33 \mathrm{E}+03 \\
\mathrm{Si} \text { value }\end{array}$ & $\begin{array}{l}2.33 \mathrm{E}+03 \\
{[17]}\end{array}$ & $\begin{array}{l}5.35 \mathrm{E}+03 \\
{[18]}\end{array}$ \\
\hline $\begin{array}{l}\text { Specific heat } \\
\text { Reference }\end{array}$ & $C_{\mathrm{sp}}=C_{\mathrm{v}} / \rho$ & $\mathrm{J} /(\mathrm{K} \mathrm{kg})$ & $\begin{array}{l}7.00 \mathrm{E}+02 \\
\text { Si value }\end{array}$ & $\begin{array}{l}7.05 E+02 \\
\text { Int }\end{array}$ & $\begin{array}{l}6.23 \mathrm{E}+02 \\
\text { Int }\end{array}$ & $\begin{array}{l}5.95 \mathrm{E}+02 \\
\text { Int }\end{array}$ & $\begin{array}{l}7.00 \mathrm{E}+02 \\
\text { Si value }\end{array}$ & $\begin{array}{l}7.13 \mathrm{E}+02 \\
{[19]}\end{array}$ & $3.20 \mathrm{E}+02$ \\
\hline $\begin{array}{l}\text { Thermal conductivity } \\
\text { Reference }\end{array}$ & $K$ & $\mathrm{~J} /(\mathrm{K} \mathrm{s} \mathrm{m})$ & $\begin{array}{l}80 \\
{[16,21]}\end{array}$ & $\begin{array}{l}10.67 \\
{[16,21]}\end{array}$ & $\begin{array}{l}5.71 \\
{[16,21]}\end{array}$ & $\begin{array}{l}5.52 \\
{[16,21]}\end{array}$ & $\begin{array}{l}80.00 \\
{[16,21]}\end{array}$ & $\begin{array}{l}1.56 \mathrm{E}+02 \\
{[18,19]}\end{array}$ & $\begin{array}{l}58.61 \\
{[18]}\end{array}$ \\
\hline Tire constant & $\tau$ & $\mathrm{s}$ & $9.00 \mathrm{E}-08$ & $7.62 \mathrm{E}-07$ & $1.40 \mathrm{E}-06$ & $1.54 \mathrm{E}-06$ & $7.44 \mathrm{E}-08$ & & \\
\hline Beam width & $b$ & $\mathrm{~m}$ & $6.60 \mathrm{E}-06$ & $6.90 \mathrm{E}-06$ & $6.48 \mathrm{E}-06$ & $6.00 \mathrm{E}-06$ & $6.00 \mathrm{E}-06$ & & \\
\hline Resonant frequency & $f=\omega / 2 \pi$ & $\mathrm{Hz}$ & 12,851 & 13,327 & 10,357 & 10,785 & 12,361 & & \\
\hline Thermoelastic $Q$-value & $Q_{\mathrm{TED}}$ & & $6.70 \mathrm{E}+05$ & $7.48 \mathrm{E}+04$ & $4.07 \mathrm{E}+04$ & $3.30 \mathrm{E}+04$ & $8.29 \mathrm{E}+05$ & & \\
\hline
\end{tabular}

The notation "Int" refers to a linear interpolation between the silicon and germanium values, based on the alloy composition. 
Theory vs. Data for MEMS Gyros from different Materials

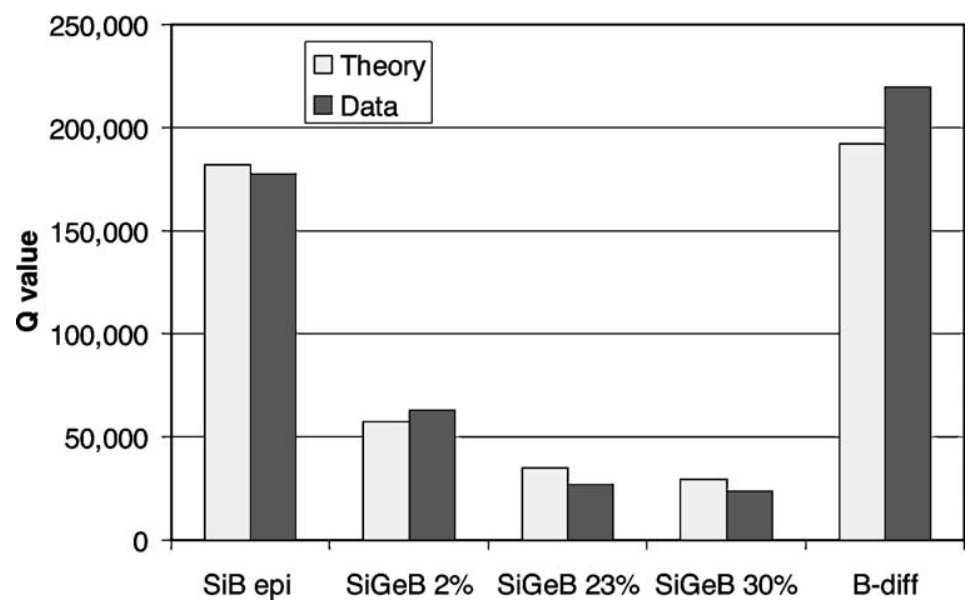

Fig. 4. Data versus calculated $Q$ values. In the theory, $Q_{\text {other }}=250,000$ is used as a fitting parameter.

material parameters used for the calculation of $Q_{\mathrm{TED}}$. We also measured devices made from Boron diffused silicon (noted as B-diff in the plots), where the Boron concentration was approximately $10^{20}$. Although the Debye resonance frequency $(1 / \tau$ in Table 1$)$ is order of magnitude above our operating frequency of about $12 \mathrm{kHz}$, the importance of the thermal conductivity is apparent when we compare the theory to our measurements.

\section{Data versus theory}

The materials tested, as summarized in Table 1, are made from: (1) silicon boron epi, (2) $\mathrm{SiGeB}$ epi, with 2 at.\% germanium, (3) SiGeB epi, with 23 at.\% germanium, (4) SiGeB epi, with 30 at.\% germanium, and (5) boron diffused silicon. We measured 9-10 devices from each wafer, and took the average $Q$-value. The standard deviation of the $Q$ measurements across a wafer was typically about $12 \%$. We compared the measured (average) $Q$ values for each material to a calculation based on the following formula:

$$
\frac{1}{Q_{\text {total }}}=\frac{1}{Q_{\text {electronics }}}+\frac{1}{Q_{\mathrm{TED}}}+\frac{1}{Q_{\text {other }}}
$$

While $Q_{\text {TED }}$ and $Q_{\text {electronics }}$ can be calculated for each material, the $Q_{\text {other }}$ represents as yet unmodeled effects such as anchor damping, any residual gas damping, and other material losses. We reason that $Q_{\text {other }}$ should be the same for all devices and materials if the calculated $Q_{\mathrm{TED}}$ accounts for all differences between the materials. Thus, we find a single value of $Q_{\text {other }}$ that minimizes the error between our set of measurements and the theory expressed by Eq. (7). The extent to which the data and theory do not match can be helpful in identifying additional material damping mechanisms. For the particular device design used in this study, $Q_{\text {other }}=250,000$ worked well for all the materials measured.
The electronics damping, calculated in the Appendix A, was in the range of $Q_{\text {electronics }}=3.5 \times 10^{11}$ but varies slightly between materials since the undercut (which we measure to be typically less than $0.5 \mathrm{~mm}$ ) can affect the readout capacitor size. The thermoelastic $Q$-value for each material is listed in Table 1. The plot in Fig. 4 compares the data to the theory.

In addition to the data presented in Fig. 4, we also compared the measured $Q$ values of various device designs. In several iterations of the tuning fork gyro, the beam widths were varied to optimize the modes of the devices. Based only on the beam width and resonance frequency data, we have been able to use thermoelastic damping to explain why some designs exhibited much higher $Q$ values than others. The match of thermoelastic damping theory to our data over a range of device designs indicates that the anchor damping does not vary much between these iterations. However, the relatively low best fit values of $Q_{\text {other }}=250,000$ suggest that anchor damping may be an important limiting factor in all designs.

\section{Conclusions}

We have measured the quality factors of resonators made from several different semiconductor materials. Our data can be explained well by thermoelastic damping and electronics damping calculations. Changes in $Q_{\mathrm{TED}}$ can affect our final measured $Q$ values by factors of two and three. Because the MEMS devices studied operate at frequencies well below the thermoelastic damping resonance, the value of $Q_{\mathrm{TED}}$ can be optimized by moving the thermoelastic Debye peak to higher frequencies. In the gyros studied, this corresponds to choosing materials with higher thermal conductivity and designing gyros with narrower flexures. In contrast, for MEMS devices, such as RF resonators and filters, the optimization of $Q_{\text {TED }}$ may require materials and designs 
that lower the thermoelastic Debye resonance, to move it further from the frequency of operation.

The simple flexural beam models provided by Zener [1,2], and Lifshitz and Roukes [4] were surprisingly successful in estimating thermoelastic $Q$ values and in identifying the material and design parameters that optimize the device $Q$. More precise optimization of complex geometries can be achieved using numerical approaches to solving the coupled thermoelastic equations. Future work in modeling additional loss mechanisms, such as anchor damping will further improve MEMS resonator designs.

\section{Acknowledgements}

The authors wish to thank Professor Gene Fitzgerald and Mathew Curie for SiGe film growth, and thanks to Draper laboratory technicians for fabrication assistance. Thanks to Robert White for his initial measurements of $Q$ factors in gyros and to Bill Sawyer for assistance with the fabrication. We especially thank Mark Mescher for valuable conversations, as well as Marc Weinberg, Chris Dubé and Richard Elliott for valuable advice and support. Finally, we thank Neil Barbour, Jim Connelly, Tony Kourepenis, and Jim Sitomer for their support of this work.

\section{Appendix A. Calculation of electronics damping}

The tuning fork resonance is excited by applying an ac voltage to the outer capacitor (left capacitor in Fig. 2). The electrostatic force on the gyro is proportional to the square of the drive voltage. At the output, the voltage bias needed to generate a readout signal can also place a force on the sensor. To model the system dynamics, we balance these electrostatic forces on the sensor with the inertia and resorting force on the masses. The force balance becomes simplest when the motion of the resonator is already decomposed into linear eigen modes. For the tuning fork mode of interest, the masses move in the wafer plane, in the $x$-direction of Fig. 2. The modal amplitude is represented by $u(t)$, and the force balance becomes

$m \ddot{u}+b \dot{u}+k u=\frac{\left(V_{\mathrm{drive}}\right)^{2}}{2} \frac{\mathrm{d} C}{\mathrm{~d} u}+\frac{\left(V_{\mathrm{n}}\right)^{2}}{2} \frac{\mathrm{d} C}{\mathrm{~d} u}$

The electrostatic forces in (6) are proportional to the change in capacitance with sensor mode amplitude. For these gyros, the drive and sense capacitors on the motor axis are comb designs, and to first order the term $\mathrm{d} C / \mathrm{d} u$ is a constant that depends on geometry but is independent of $u$. In Eq. (6), $m$ is the modal mass and $b$ is the modal damping from sources other than the electronics.

If the amplifier gain is infinite, the node voltage $V_{\mathrm{n}}$ is equal to $V_{\mathrm{B}}$. The gain is assumed to be purely imaginary at the operating frequency. For finite gain, we write

$V_{\mathrm{n}}=V_{\mathrm{B}}-I_{\mathrm{s}} R_{\text {in }}$

$I_{\mathrm{s}}=\dot{C} V_{\mathrm{n}}$

$R_{\text {in }}=\frac{1}{\omega C_{\mathrm{f}}|A|}$

$I_{\mathrm{s}}$ is the sensor current, $R_{\text {in }}$ the input resistance of the op-amp, and $A$ the amplifier gain. These equations combine to give

$V_{\mathrm{n}}=\frac{V_{\mathrm{B}}}{1+\dot{u}(\mathrm{~d} C / \mathrm{d} u) R_{\mathrm{in}}}$

For large amplifier gain, $R_{\mathrm{in}}$ is small and a Taylor expansion can be applied to (10). In the limit of large amplifier gain, Eq. (6) becomes

$m \ddot{u}+b \dot{u}+k u=\frac{\left(V_{\mathrm{drive}}\right)^{2}}{2} \frac{\mathrm{d} C}{\mathrm{~d} u}+\frac{\left(V_{\mathrm{B}}\right)^{2}}{2} \frac{\mathrm{d} C}{\mathrm{~d} u}-\left(V_{\mathrm{B}}\right)^{2} \frac{\mathrm{d} C}{\mathrm{~d} u} R_{\mathrm{in}} \dot{u}$

Thus, the applied bias results in a term proportional to velocity. By taking the ratio of stored energy to the energy dissipated per cycle, the contribution of the electronics to the $Q$ of the system is

$Q_{\text {electronics }}=\frac{m C_{\mathrm{f}}|A| \omega^{2}}{2 V_{\mathrm{B}}^{2}(\mathrm{~d} C / \mathrm{d} u)^{2}}$

Note that designing for large readout gains (high $V_{\mathrm{B}}$ and $\mathrm{d} C / \mathrm{d} u$ ) can degrade the resonator $Q$-value. However, the readout amplifier gain can compensate for this effect.

\section{References}

[1] C. Zener, Phys. Rev. 52 (1937) 230.

[2] C. Zener, Phys. Rev. 53 (1938) 90.

[3] T.V. Roszhart, in: Proceedings of the Solid-State Sensor and Actuator Workshop, Hilton Head Island, SC, IEEE, New York, 1990, p. 13.

[4] R. Lifshitz, M. Roukes, Phys. Rev. B 61 (8) (2000) 61.

[5] K. Yasumura, T. Stowe, E. Chow, T. Pfafman, T. Kenny, B. Stipe, D. Rugar, J. Microelectromech. Syst. 9 (1) (2000) 117.

[6] K.A. Gibbons, J.T. Borenstein, D.S. Nokes, M.S. Weinberg, The design, fabrication, and testing of a micromechanical silicon oscillating accelerometer, AIAA Guidance, Navigation, and Control Conference and Exhibit, Boston, MA, 10-12 August 1998, Collection of Technical Papers, Pt. 2 (A98-37001 10-63), 1998, pp. 12961306.

[7] A. Kourepenis, J.T. Borenstein, J. Connelly, R. Elliott, P. Ward, M.S. Weinberg, Performance of MEMS inertial sensors, in: Proceedings of the IEEE 1998 Position Location and Navigation Symposium, vol. 1, Palm Springs CA, 1998.

[8] M.S. Weinberg, B.T. Cunningham, C.W. Clapp, Modeling flexural plate wave devices, J. Microelectromech. Syst. 9 (3) (2000) 370-379.

[9] Kun Wang, Yinglei Yu, Ark-Chew Wong, Clark T.-C. Nguyen, VHF free-free beam high- $Q$ micromechanical resonators, Technical Digest, Proceedings of the 12th International IEEEE Micro Electro Mechanical Systems Conference, Orlando, FL, 1999, pp. 453-458.

[10] J. Dismukes, L. Ekstrom, E. Steigmeier, I. Kudman, D. Beers, J. Appl. Phys. 35 (10) (1964). 
[11] Varghese, Amantea, Sauer, Senturia, Resistive Damping of pulsesensed capacitive position sensors, in: Proceedings of IEEE Transducers '97, Chicago, IL, 1997, p. 1121.

[12] E.M. Lifshitz, L.D. Landau, Theory of Elasticity, Pergamon Press, Oxford, 1959

[13] W. Nowacki, Thermoelasticity, 2nd ed., Pergamon Press, Oxford, 1986 (Chapter 1).

[14] R. Lakes, Viscoelastic Solids, CRC Press, Boca Raton, 1999, pp. 294-297.

[15] J.T. Borenstein, N.D. Gerrish, M.T. Currie, E.A. Fitzgerald, A new ultra-hard etch-stop layer for high precision micromachining, in: Proceedings of 12th International Workshop on Micro Electro Mechanical Systems-MEMS, IEEE Robotics and Automation Society, Orlando, FL, 1999, pp. 205-210.
[16] K.L. Wang, X. Zheng, Thermal properties of SiGe, in: E. Kasper (Ed.), Properties of Strained and Relaxed Silicon Germanium, INSPEC, 1995.

[17] Y. Okada, Diamond cubic Si: structure, lattice parameter and density, in: R. Hull (Ed.) The Properties of Crystalline Silicon, INSPEC, 1999.

[18] http://www.webelements.com.

[19] H.-M. Kagaya, T. Soma, Specific Heats of C-Si and molten Si, in: R. Hull (Ed.) The Properties of Crystalline Silicon, INSPEC, 1999.

[20] C. Cabuz, K. Fukatsu, T. Kurabayashi, K. Minami, M. Esashi, Microphysical investigations on mechanical structures realized in $\mathrm{p}+$ silicon, J. Microelectromech. Syst. 4 (3) (1995) 109-118.

[21] J. Dismukes, L. Ekstrom, E. Steigmeier, I. Kudman, D. Beers, Thermal and electrical properties of heavily doped Ge-Si alloys up to 1300 K, J. Appl. Phys. 35 (10) (1964). 